\title{
Cognitive decline in Parkinson's disease: the impact of the motor phenotype on cognition
}

\author{
Jennifer Wojtala, ${ }_{1}^{1,2}$ Ines Ann Heber, ${ }_{1}^{1}$ Petra Neuser, ${ }_{1}^{3}$ Julia Heller, ${ }^{1,2}$ Elke Kalbe, ${ }^{4}$ \\ Sarah P Rehberg, ${ }^{4}$ Alexander Storch, ${ }^{5,6}$ Katharina Linse, ${ }^{5}$ Christine Schneider ${ }^{5}$ \\ Susanne Gräber, ${ }^{7}$ Daniela Berg, ${ }^{7,8}$ Judith Dams, ${ }^{9}$ Monika Balzer-Geldsetzer, ${ }^{9}, 10$ \\ Rüdiger Hilker-Roggendorf, ${ }_{1}^{4}$ Carola Oberschmidt, ${ }_{1}^{11}$ Simon Baudrexel, ${ }^{11}$ Karsten Witt, ${ }^{12}$ \\ Nele Schmidt, ${ }^{8}$ Günther Deuschl, ${ }^{7}$ Brit Mollenhauer, ${ }_{13}^{13}$ Claudia Trenkwalder, ${ }^{13}$ \\ Inga Liepelt-Scarfone, ${ }^{7}$ Annika Spottke, ${ }^{14}$ Sandra Roeske, ${ }^{14}$ Ullrich Wüllner, ${ }^{14}$ \\ Hans-Ulrich Wittchen, ${ }^{15,16}$ Oliver Riedel, ${ }^{17}$ Richard Dodel, ${ }^{9,10}$ Jörg B Schulz, ${ }^{1,2}$ \\ Kathrin Reetz ${ }^{1,2}$
}

For numbered affiliations see end of article.

\section{Correspondence to} Professor Kathrin Reetz, Department of Neurology, RWTH Aachen University, Aachen, 52074, Germany; kreetz@ukaachen.de

Received 13 June 2018 Revised 26 July 2018 Accepted 17 August 2018 Published Online First 8 October 2018

Check for updates

(C) Author(s) (or their employer(s)) 2019. No commercial re-use. See rights and permissions. Published by BMJ.

To cite: Wojtala J, Heber IA, Neuser $P$, et al. J Neurol Neurosurg Psychiatry

2019:90:171-179.

\section{ABSTRACT}

Objectives Parkinson's disease (PD) is the second most common neurodegenerative disorder and is further associated with progressive cognitive decline. In respect to motor phenotype, there is some evidence that akinetic-rigid PD is associated with a faster rate of cognitive decline in general and a greater risk of developing dementia.

The objective of this study was to examine cognitive profiles among patients with PD by motor phenotypes and its relation to cognitive function.

Methods Demographic, clinical and neuropsychological cross-sectional baseline data of the DEMPARK/ LANDSCAPE study, a multicentre longitudinal cohort study of 538 patients with PD were analysed, stratified by motor phenotype and cognitive syndrome. Analyses were performed for all patients and for each diagnostic group separately, controlling for age, gender, education and disease duration.

Results Compared with the tremor-dominant phenotype, akinetic-rigid patients performed worse in executive functions such as working memory (Wechsler Memory Scale-Revised backward; $p=0.012)$, formallexical word fluency $(p=0.043)$, card sorting $(p=0.006)$, attention (Trail Making Test version $A ; p=0.024$ ) and visuospatial abilities (Leistungsprüfungssystem test 9; $p=0.006)$. Akinetic-rigid neuropsychological test scores for the executive and attentive domain correlated negatively with non-tremor motor scores. Covariateadjusted binary logistic regression analyses showed significant odds for PD-mild cognitive impairment for not-determined as compared with tremor-dominant $(O R=3.198)$ and akinetic-rigid PD $(O R=2.059)$. The odds for PD-dementia were significant for akineticrigid as compared with tremor-dominant phenotype (OR=8.314).

Conclusion The three motor phenotypes of PD differ in cognitive performance, showing that cognitive deficits seem to be less severe in tremor-dominant PD. While these data are cross-sectional, longitudinal data are needed to shed more light on these differential findings.

\section{INTRODUCTION}

Parkinson's disease (PD) is the second most common neurodegenerative disorder and mainly characterised by its motor symptoms of tremor, bradykinesia, rigidity and postural instability. During the last years, it has increasingly been acknowledged that non-motor symptoms, such as dementia, depression or sleep disorders, are also common features. ${ }^{1}$ Cognitive decline in PD includes several cognitive domains and presents one of the most debilitating manifestations. ${ }^{2}$

There is evidence that approximately 30\%-40\% of patients with PD develop cognitive deficits and may get dementia (PD-D), starting with mild cognitive impairment (PD-MCI) and evolving to a more generalised dysfunction of cognition. ${ }^{3}$

Differences in cognitive profiles - especially executive problems, ${ }^{4}$ assumed to be caused by dopaminergic dysfunction in the frontostriatal circuit - are prominent ${ }^{5}$ and PD is further associated with diminished spatial and verbal working memory skills. ${ }^{6}$

PD can be subclassified into three motor subtypes $^{7}$ : tremor-dominant (TR-D), akinetic-rigid, exhibiting postural instability and gait difficulty (PIGD-D) and 'not-determined' (ND), with patients showing mixed symptoms (phenotype abbreviations are used for tables/figures). Whereas the TR-D type is characterised by a resting or postural tremor, patients with akinetic-rigid PD mainly suffer from bradykinesia, akinesia and/or rigidity. ${ }^{8}$ Akinetic-rigid patients have previously been associated with faster cognitive decline, greater risk of dementia and higher sensitivity for depression. ${ }^{9}$

One assumption is that akinetic-rigid patients suffer from deficits in the cortico-striatal-thalamic loop, while TR-D patients have deficits in the cerebellar-thalamic-cortical circuit. ${ }^{7}$

Emphasising the diversity of cognitive profiles in PD, studies for example showed a significant relation between working memory deficits in akinetic-rigid but not TR-D PD. ${ }^{10}$ This association between motor deficits and working memory impairments may be due to a dopamine denervation in frontal cortical and striatal areas, emphasised by 
dopaminergic treatment showing enhancements in spatial and verbal working memory. ${ }^{11}$

Patients who have been diagnosed as TR-D tend to progress to the akinetic-rigid type in the course of PD. ${ }^{12}$ This theory is in line with research assuming that the transition can be related to the progressive PD pathology, with motor symptoms initially being limited to brainstem functions (as in TR-D PD) to a broader pathology influencing other cortical areas and brainstem regions (as in akinetic-rigid PD). ${ }^{13}$ Anyhow, there are inconsistencies among previous research results, particularly with respect to cognitive function and its association with motor subtypes. Therefore, proper diagnosis and examination of cognitive impairments are essential for patient care.

Although there is already some evidence, there is a growing interest and need for a detailed understanding of the subtypes, influencing factors on the individual progression and staging in PD. In contrast to previous studies, we aim at identifying distinct neuropsychological characteristics with respect to the motor phenotype (TR-D, PIGD-D, ND) in the entire group of patients with $\mathrm{PD}$ as well as within each diagnosis group (patients with a normal cognitive profile (PD-N), PD-MCI and PD-D), which could help identify a baseline profile and allows predictions about incidence of cognitive impairment and its possible association to motor phenotype. The investigations were performed within the DEMPARK/LANDSCAPE study, ${ }^{14}$ a large German cohort study on cognitive functions in PD.

\section{METHODS}

\section{The DEMPARK/LANDSCAPE database}

The DEMPARK/LANDSCAPE study is a multicentre longitudinal cohort study of patients with PD that aims at documenting the progression of cognition in PD and identifying risk factors for the development of MCI and dementia. Patients have been recruited ( $n=625$ at baseline) in eight movement disorder clinics within university hospitals across Germany (Aachen $n=104$, Bonn $\mathrm{n}=44$, Dresden $\mathrm{n}=82$, Frankfurt/Main $\mathrm{n}=83$, Kassel $n=60$, Kiel $n=77$, Marburg $n=82$, Tübingen $n=93$ ). They were assessed annually in each clinic with a comprehensive clinical and neuropsychological test battery. ${ }^{14}$

\section{Participants}

Inclusion criteria for the DEMPARK/LANDSCAPE cohort included an age between 45 and 80 years and a diagnosis of idiopathic PD according to the UK Parkinson's Disease Society Brain Bank clinical diagnostic criteria. ${ }^{15}$ As also patients with Lewy body dementia according to the criteria by McKeith ${ }^{16}$ were included in the cohort study, it is important to note that this work only focuses on patients with idiopathic PD. After the baseline visit the cohort was further divided into three subgroups: PD-N, PD-MCI, which was defined according to the criteria by Petersen, ${ }^{17}$ and PD-D who were diagnosed according to the consensus guidelines by Emre et $a l,{ }^{18}$ operationalised by Dubois et al. ${ }^{19}$ Patients with PD with a validated diagnosis and phenotype, aged 50 or above and at least 8 years of education were considered for analysis $(n=538)$.

\section{Clinical assessment}

For the clinical assessment, patients were examined by a neurologist. Data collected on the first visit included initial manifestation and duration of symptoms, date of initial diagnosis, Hoehn $\&$ Yahr state and medication. Furthermore, patients underwent a physical and neurological examination. Motor symptoms were assessed by the Unified Parkinson's Disease Rating Scale
(UPDRS parts I, III, IV; https://www.movementdisorders.org). Additionally, the Neuropsychiatric Inventory, ${ }^{20}$ the short form of the Geriatric Depression Scale (GDS), ${ }^{21}$ the Parkinson's Disease Questionnaire 39-item version (PDQ-39) ${ }^{22}$ and the EuroQol-5 Dimension questionnaire (EQ-5D, https://euroqol.org) were administered to assess quality of life, symptoms of depression, neuropsychiatric symptoms and limitations in activities of daily life.

\section{Cognitive assessment}

This work examines data collected within the patients' initial DEMPARK baseline visit. All neuropsychological assessments were carried out by trained neuropsychologists. The neuropsychological test battery included two screening tests, the Mini-Mental State Examination (MMSE) and the Parkinson Neuropsychometric Dementia Assessment (PANDA), ${ }^{23}$ as well as a set of standardised tests for the assessment of different cognitive domains.

Verbal memory was tested by the Consortium to Establish a Registry for Alzheimer's Disease (CERAD) subscores of word list learning, delayed recall and recognition. Non-verbal memory was assessed by CERAD delayed recall of copied figures. Attentional functions were assessed by a Stroop interference task (Farb-Wort-Interferenztest), ${ }^{24}$ the Trail Making Test version A (TMT-A $)^{25}$ and the Brief Test of Attention. ${ }^{26}$ Executive functions were tested by phonemic and semantic verbal fluency subtests of the CERAD + battery (a version of the CERAD, which adds phonemic verbal fluency and the Trail Making A and B), ${ }^{27}$ as well as the TMT-B/A index and the Modified Card Sorting Test. ${ }^{28}$ Accordingly, visuospatial functions were assessed by the figure copy task of the CERAD, as well as the subtests LPS-7 (mental rotation) and LPS-9 (spatial imagination) of the German 'Leistungsprüfsystem' $50+\left(\right.$ LPS 50+). ${ }^{29}$ Language abilities were assessed by the shortened version of the Boston Naming Test within the CERAD + battery. The recognition subtest of the CERAD as well as the TMT-A were not considered for diagnosis classification.

\section{Operationalisation of TR-D, PIGD-D and ND}

The UPDRS III scores built the basis for the motor phenotype classification and were derived as follows. ${ }^{30}$ The tremor score was derived by the sum of UPDRS items 20 (resting tremor) and 21 (postural tremor of hands) divided by 7 (number of subitems). The non-tremor score was calculated by summing up UPDRS scores of items 18 (speech), 19 (face expressions), 22 (rigidity), 27 (arising from chair), 28 (posture), 29 (gait), 30 (postural stability) and 31 (bradykinesia/hypokinesia of the body) divided by 12 (number of subitems). TR-D was classified by a ratio (tremor score/non-tremor score) $\geq 1.5$ or a positive tremor score and a 0 non-tremor score $(n=40)$, akinetic-rigid by a ratio $\leq 1.0$ or a 0 tremor score and a positive non-tremor score $(n=455)$. For ND the ratio ranged from $>1$ to $<1.5$ or both the tremor and non-tremor scores were $0(n=43)$.

\section{Statistical analyses}

Statistical data analyses were carried out by a central statistician (PN) using SAS V.9.4 (SAS Institute) at the coordination centre for clinical studies (Koordinierungszentrum für Klinische Studien), University of Marburg, Germany. Tests were two sided; except for the correlation analyses, in which the alpha level was set to 0.01 for each test, an alpha level of 0.05 was used throughout all other analyses without adjustment for multiple testing. Differences in demographic, clinical and neuropsychological 
characteristics and screening tools were tested between motor phenotypes for the entire group of patients with PD and for each diagnosis subgroup separately, controlling for age at baseline, gender, years of education and disease duration. Here, the PD-D subgroup needed to be omitted from comparing phenotypes as it consisted almost exclusively of akinetic-rigid patients $(95,89 \%)$. Using the age, education and gender-corrected normative scores (Z scores) of the CERAD + battery, ${ }^{27}$ explicit adjustment was additionally necessary for disease duration. For age-corrected $\mathrm{T}$ scores, additional covariate adjustment was performed with regard to the same variables. After adjustments, $n=538$ patients were included into analyses.

Differences in continuous covariates were tested applying the Kruskal-Wallis test and Dunn's test for multiple post hoc comparisons. Differences in gender were tested with the $\mathrm{X}^{2}$ test. As rank analysis of covariance (ANCOVA) is limited to comparison of groups with similar distributions of covariates, ANCOVA was applied in the entire group of patients with PD and the PD-N subgroup, exhibiting significant differences in disease duration and education between phenotypes. Given the similar distributions of covariates in the PD-MCI subgroup, rank ANCOVA was applied if the normality assumption of residuals was violated by using the Cochran-Mantel-Haenszel mean score statistic, and ANCOVA was performed otherwise. The Tukey-Kramer test was used for multiple post hoc comparisons.

To investigate the relationship between neuropsychological tests and motor symptoms, the Spearman partial correlation coefficient-controlling for age, gender, education and disease duration-was determined between neuropsychological and tremor and non-tremor scores in the entire PD group. In this context, correlations ranging from $0.0<|\mathrm{r}|<0.3$ were considered as weak, $0.3<|\mathrm{r}|<0.7$ were considered as moderate and correlations $|\mathrm{r}|>0.7$ were considered as strong. ${ }^{31}$

In order to analyse inclusion into a diagnosis group depending on phenotype, covariate-adjusted binary logistic regressions were executed with diagnosis as dependent variable and phenotype as independent variable.

\section{RESULTS}

\section{Clinical and cognitive parameters: motor phenotype}

Age, education and gender distributions were comparable in each phenotype of the total cohort (see table 1). Differences between akinetic-rigid and TR-D PD for the whole group of patients were observed, showing that TR-D patients had a shorter disease duration $(\mathrm{p}=0.003)$, fewer symptoms of depression (GDS-15: $\mathrm{p}=0.002$ ) and better quality of life (PDQ-39: $\mathrm{p}=0.003$; EQ-5D: $\mathrm{p}=0.005)$. Moreover, they differed regarding state (Hoehn \& Yahr; $\mathrm{p}=0.002$ ) and in symptoms, with lower UPDRS scores (UPDRS III: $p=0.042$; bradykinesia: $p<0.001$; rigidity: $\mathrm{p}<0.001)$. Significant differences in resting tremor score were present between ND and akinetic-rigid type $(\mathrm{p}<0.001)$ and TR-D and akinetic-rigid type $(\mathrm{p}<0.001)$, revealing higher scores for ND and TR-D compared with akinetic-rigid patients. Action tremor scores differed significantly between all phenotypes with highest score for TR-D type compared with ND $(p=0.005)$ and akinetic-rigid type $(\mathrm{p}<0.001)$.

Analyses of the short cognitive tests (table 2) revealed marked results for the PANDA, but not for the MMSE, showing better performance of ND compared with akinetic-rigid $\mathrm{PD}(\mathrm{p}=0.003$, figure 1).

Comparing the different cognitive domains regarding phenotype, the following distinct differences can be reported (table 2, figure 1). In the domain of memory functions, significant differences were present for digit span forward, showing better scores for ND compared with akinetic-rigid PD $(p=0.033)$. Additionally, significant differences for the backward condition were also present, in which both ND $(\mathrm{p}=0.044)$ and TR-D patients $(\mathrm{p}=0.012)$ scored better compared with akinetic-rigid ones. In terms of word fluency, the ND type performed better in semantic word fluency compared with the akinetic-rigid type $(\mathrm{p}=0.015)$, while TR-D patients were better in word production of formal-lexical word fluency compared with akinetic-rigid PD $(p=0.043)$. Additionally, card sorting was better in TR-D compared with akinetic-rigid patients $(p=0.006)$. In the cognitive domain of attention, both the ND type $(p=0.022)$ and the TR-D type $(\mathrm{p}=0.024)$ showed faster information processing in TMT-A than the akinetic-rigid one. Furthermore, in the visuospatial task (LPS-9) TR-D patients showed superior performance to that of akinetic-rigid patients $(\mathrm{p}=0.006)$.

\section{Clinical and cognitive parameters: diagnostic subgroups}

When analysing the diagnosis groups separately, we found that within (1) the PD-N group differences between akinetic-rigid and TR-D patients were present for education $(\mathrm{p}<0.001)$, showing that the TR-D type had lower education levels. Furthermore, differences between TR-D and ND $(p=0.011)$ and TR-D and akinetic-rigid PD $(p=0.020)$ were observed for the GDS-15, indicating fewer symptoms of depression for the TR-D type. TR-D patients were faster in test performance in TMT-A than akinetic-rigid patients $(p=0.021)$. Next to that, the major findings regarding the motor phenotype classification were that TR-D patients also presented lower scores compared with akinetic-rigid ones in Hoehn \& Yahr $(p=0.026)$, bradykinesia $(p=0.003)$ and rigidity score ( $\mathrm{p}=0.014$; see table 2 for more details).

(2) Within the PD-MCI group, differences were found for both screening tests, showing better scores for ND compared with akinetic-rigid patients in the MMSE $(\mathrm{p}=0.004)$ and the PANDA, illustrating better test results in the ND group compared with TR-D $(\mathrm{p}=0.038)$ and akinetic-rigid patients $(\mathrm{p}=0.002)$.

Additionally, differences were present for the forward condition of the digit span test, showing that the ND group had, in contrast to the akinetic-rigid group, a better ability to recall longer sequences of numbers $(\mathrm{p}=0.007)$.

Moreover, the classification of phenotype was confirmed statistically as differences between TR-D PD and akinetic-rigid PD in terms of lower bradykinesia $(p=0.008)$, lower rigidity $(\mathrm{p}=0.021)$, higher resting $(\mathrm{p}<0.001)$ and action tremor $(\mathrm{p}<0.001)$ were present (table 2$)$.

\section{Correlation analysis}

The correlation analysis of neuropsychological data and non-tremor scores in the entire group revealed weak correlations for akinetic-rigid patients: the PANDA showed a negative correlation with non-tremor scores $(\mathrm{r}=-0.21940, \mathrm{p}<0.001)$. Further negative correlations with non-tremor scores were present in semantic word fluency $(\mathrm{r}=-0.16075, \mathrm{p}<0.001)$. Also, correlations of tests measuring attention were highly significant for the non-tremor measures (Stroop word reading: $\mathrm{r}=-0.19511, \mathrm{p}<0.001$; Stroop colour naming: $\mathrm{r}=-0.21841$, $\mathrm{p}<0.001$; Stroop interference: $\mathrm{r}=-0.19553, \mathrm{p}<0.001$ ). In the visuospatial domain, a significant correlation between the non-tremor measures and LPS-9 was observed $(r=-0.17007$, $\mathrm{p}<0.001)$.

\section{Binary logistic regression}

ORs for cognitive impairment (PD-MCI, PD-D) versus no cognitive impairment (PD-N) depending on motor phenotype were 


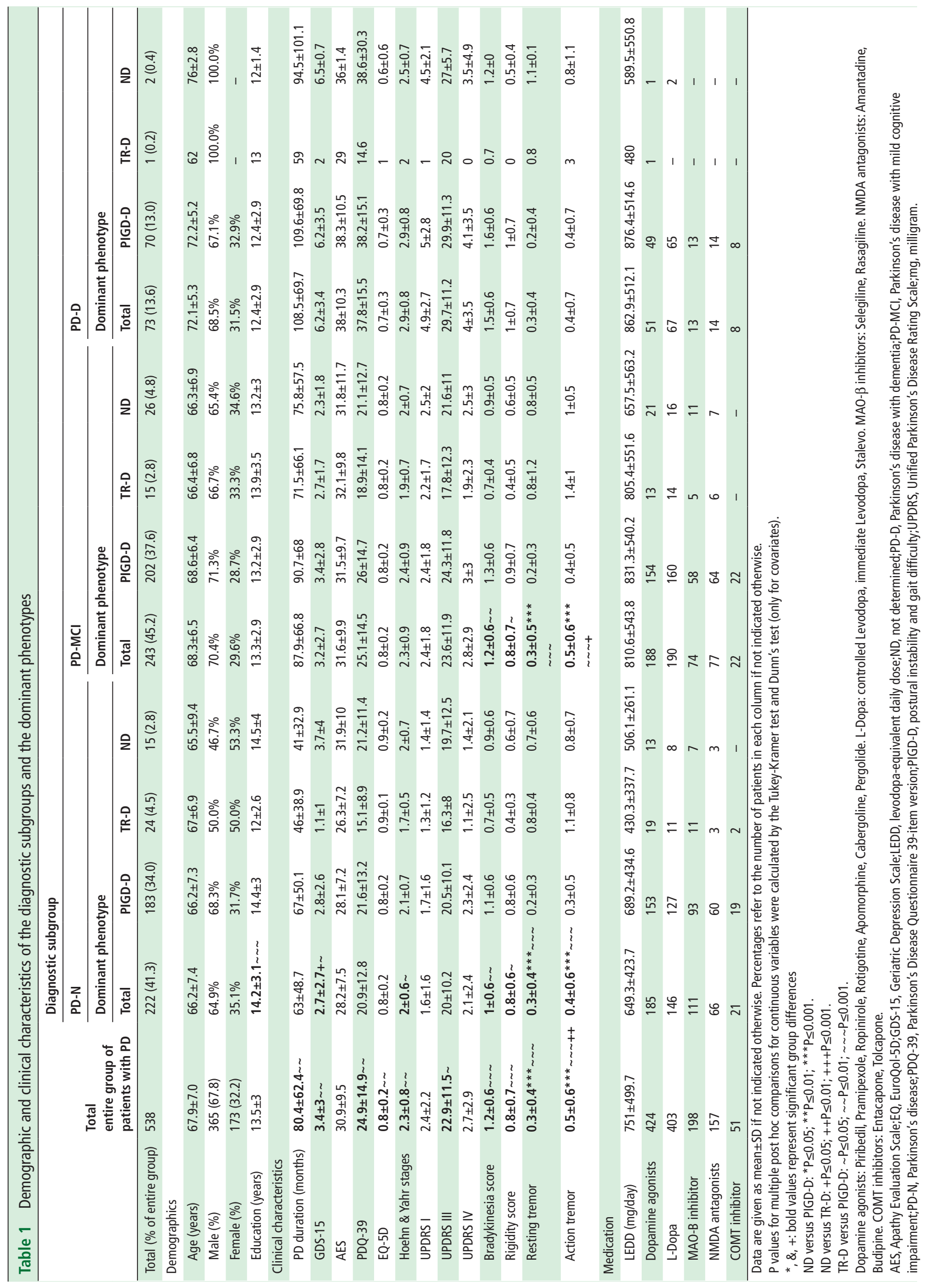




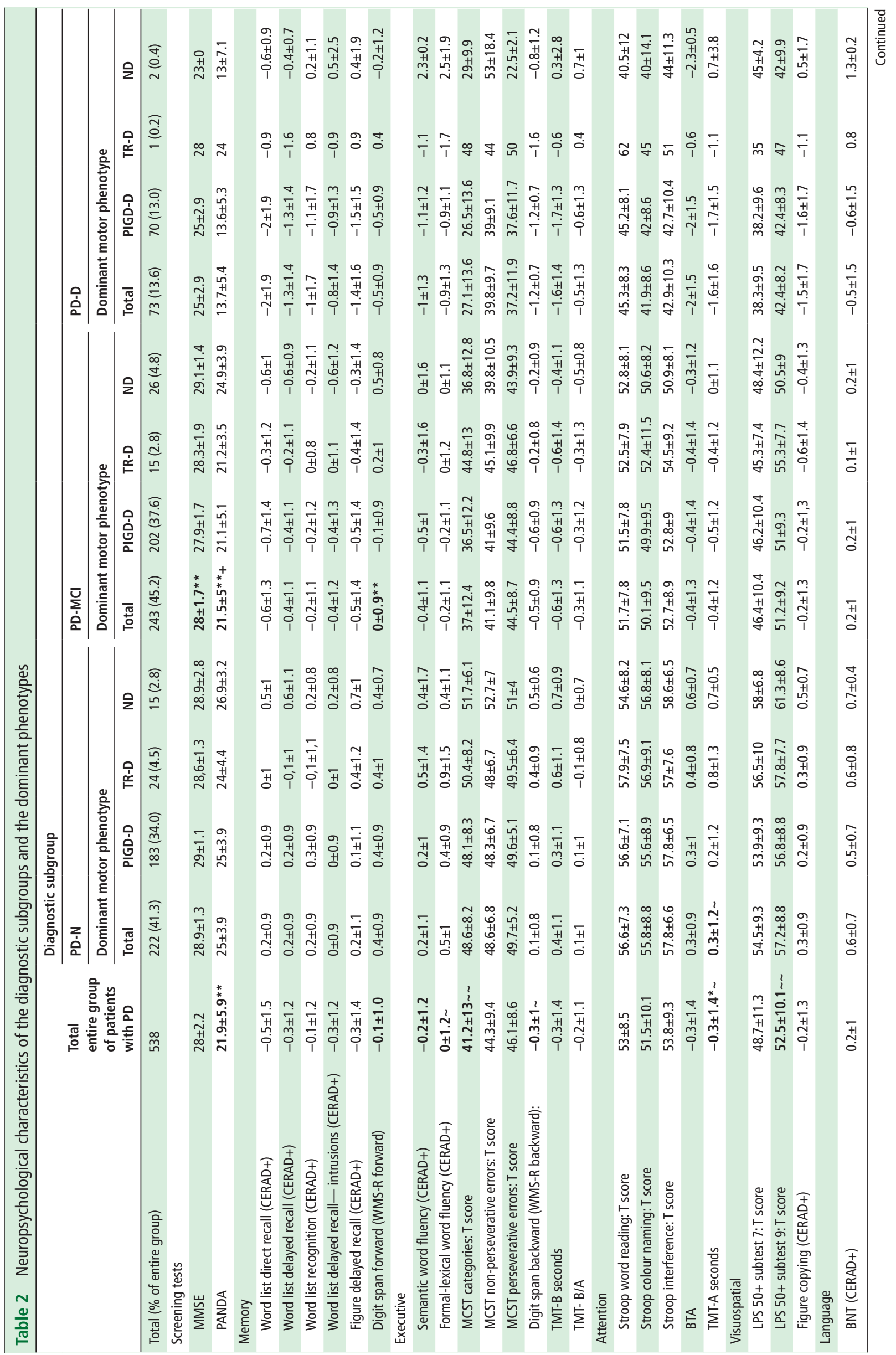


calculated by binary logistic regressions adjusted for age, education, gender and disease duration. The odds for developing MCI in the ND subgroup were three times higher than in the TR-D group (OR 3.198; 95\% CI 1.233 to 8.293; $\mathrm{p}=0.017$ ) and twice as high compared with the akinetic-rigid subgroup (OR 2.059; $95 \%$ CI 1.016 to $4.173 ; \mathrm{p}=0.045$ ). The odds for dementia in akinetic-rigid PD were eight times higher than in TR-D PD (OR $8.314 ; 95 \%$ CI 1.023 to $67.578 ; p=0.048)$. Furthermore, the ability of the motor phenotype to discriminate between levels of cognitive impairment was assessed by the area under the receiver operating characteristic curve (AUC, figure 2). Although both AUCs were shown to differ significantly from random guessing (AUC $=0.5$; both $\mathrm{p}<0.0001$ ), discrimination between PD-N and PD-MCI (AUC $=0.6835$, figure $2 \mathrm{~A}$ ) was worse than between PD-N and PD-D (AUC=0.8365, figure 2B).

\section{DISCUSSION}

In addition to previous studies we identified for the first time distinct neuropsychological characteristics with respect to the motor phenotype in the entire group of patients with $\mathrm{PD}$ as well as within each diagnosis group (PD-N, PD-MCI, PD-D). Akinetic-rigid patients show greater cognitive impairment as compared with TR-D patients, which is in line with previous studies. ${ }^{9}$ TR-D patients, however, scored lower in cognitive tests such as digit span, word fluency and attention as compared with ND patients. Executive functions seemed to be most impaired in akinetic-rigid PD, followed by attention and memory.

Furthermore, we found that visuospatial abilities were also affected in akinetic-rigid patients. Test scores measuring word fluency, attention and visuospatial functions showed slightly negative correlations with UPDRS non-tremor scores, from which phenotype classification was derived. Especially the executive and attentive domains correlated with these scores, which again match current literature claiming that motor symptoms have an impact on cognition, ${ }^{932}$ although these weak correlations have to be interpreted with caution. A recent review on cognitive changes in prodromal PD specifically reported the executive cognitive domain to be impaired in patients with prodromal PD, suggesting that the respective changes may be considered as a marker of disease progression and cognitive decline, ${ }^{33}$ which is not surprising as executive functions are affected by frontal lobe regions and are greatly sensitive to synucleinopathies.

Finally, the odds for PD-D versus PD-N were estimated to be eight times higher for the akinetic-rigid than for the TR-D type, indicating that akinetic-rigid patients have a higher risk to develop dementia. In summary, our data demonstrate that cognitive profiles differ substantially for the motor phenotypes. ${ }^{932}$

Contradictory to current literature, ${ }^{34}$ we observed memory impairment only for one neuropsychological test (digit span forward) and mainly in patients with PD-MCI. As already outlined, this could be caused by different cognitive pathways resulting in different cognitive profiles. The 'dual syndrome hypothesis' suggests that deficits in executive functions are related to a dopamine depletion in the frontostriatal circuit, while visuospatial and memory deficits are more linked to a depletion of choline in the posterior loop. ${ }^{35}$ In this context, the frontostriatal loop, transmitting from the dorsal striatum to the dorsolateral prefrontal cortex, may be affected earlier in PD. This might indicate that, while posterior cortical disturbances are related to a more rapid progression of dementia, patients primarily exhibiting executive disturbances remain more stable over time. ${ }^{36}$ This hypothesis is also corroborated by our findings, 


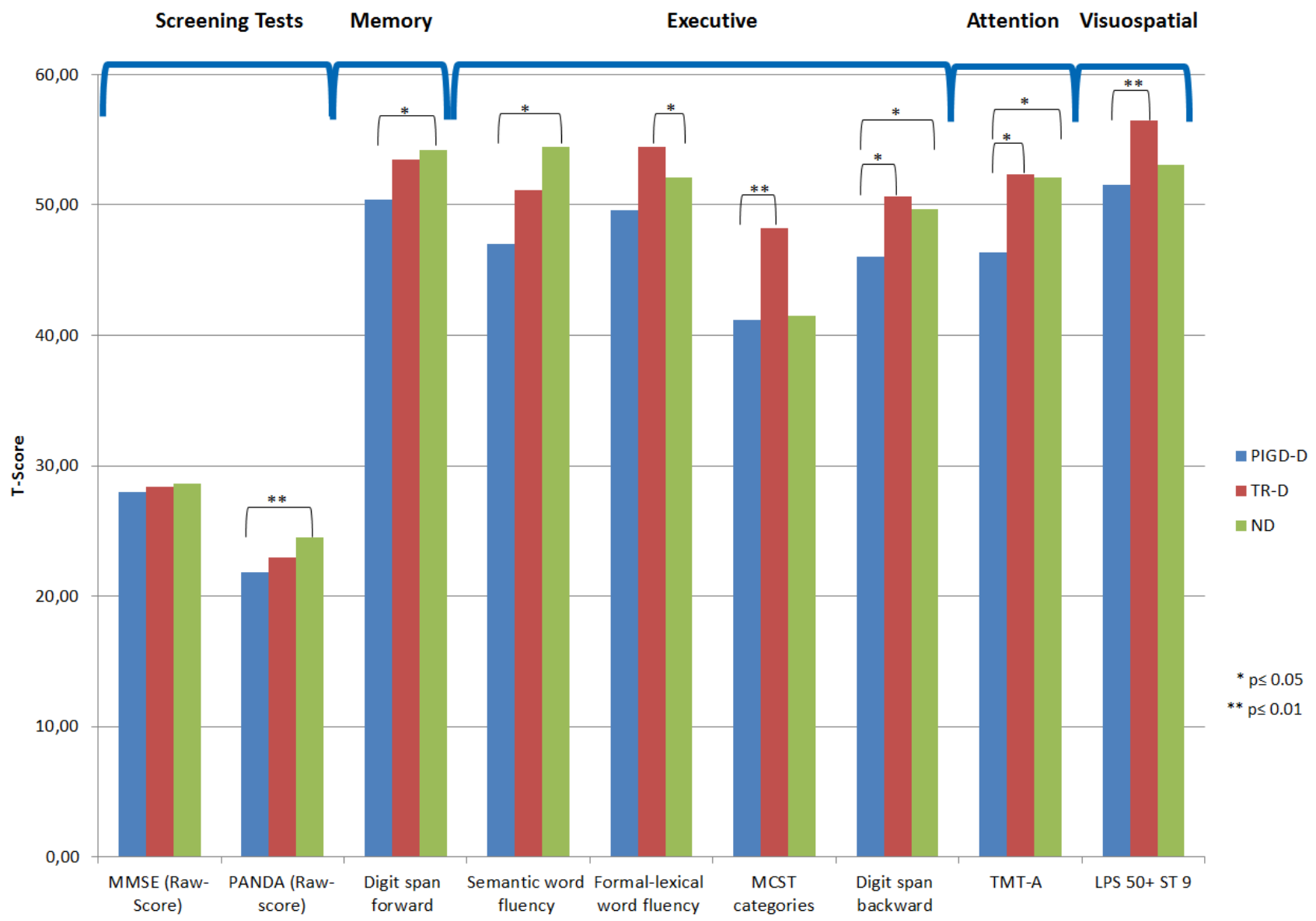

Figure 1 Differences between motor phenotypes in the neuropsychological test battery for the total group. For better visualisation, the test scores of the different neuropsychological tests were converted into T scores if not indicated otherwise. ND, not-determined; PIGD-D, akinetic rigid; TR-D, tremordominant. LPS 50+, Leistungsprüfsystem for aged 50+ (subtest 7: spatial rotation, subtest 9: spatial imagination); MCST, Modified Card Sorting Test; MMSE, Mini-Mental State Examination; PANDA, Parkinson Neuropsychometric Dementia Assessment; TMT, Trail Making Test.

as frontostriatal circuits are part of executive functions, which were shown to be impaired.

Concerning the different phenotypes, akinetic-rigid PD had more difficulties in word fluency, working memory and sorting abilities compared with TR-D and ND patients. Apart from this, ND patients scored significantly better in formal-lexical word fluency as compared with TR-D patients. Although essential tremor (ET) results from a different pathology, a non-direct parallel can be drawn, as these impairments have also been found in patients with ET, with more difficulties in lexical as compared with semantic word fluency. ${ }^{37}$

Investigating differences in phenotypes within a diagnosis group, we found distinctions in digit span forward in the total and the MCI group, indicating a decline in memory performance. Further, distinctions in TMT-A in PD-N and total group indicated an impairment in attention/speed of processing already present in patients with PD with normal cognition. However, it should be mentioned that these differences might also be caused by motor-driven deficits. ${ }^{38}$ Furthermore, the MMSE seems to be less efficient in detecting cognitive changes ${ }^{39}$ (only in PD-MCI). The PANDA, however, was able to detect cognitive impairment in PD in general, being particularly sensitive in PD-MCI. This screening tool might therefore be well suited to monitor disease progression in a clinical setting. This may also result from its uniqueness for testing cognitive functions that are especially impaired in patients with PD. Thus, a crucial challenge will be to adopt standard cognitive tests for detection of cognitive changes already at an early stage.

As it has been speculated that TR-D patients tend to progress to the akinetic-rigid type in the course of $\mathrm{PD},{ }^{12}$ we controlled for disease duration. Interestingly, the proportion of TR-D patients in the PD-D group is very small and one could thus assume that the quantity of TR-D patients diminishes as the disease progresses. Because classification of phenotype was done at baseline, a transition and resulting influence on cognition needs to be addressed in future analyses.

Contrary to our expectations that ND PD is a state in between TR-D and akinetic-rigid PD, we found results indicating better scores for this group for some measures. These results are not in line with a stage-dependent progression. ${ }^{12} \mathrm{~A}$ potential explanation might be that our patients were 'fitter' compared with the other phenotypes. Additionally, this group had lower medication doses, reported a better quality of life and lower depression scores. Furthermore, it also highlights the heterogeneity of the disease, as transition might not occur linear and there might be different biological and pathophysiological mechanisms. At this juncture an interpretation is difficult, as it might be part of the transition process, ${ }^{12}$ thus longitudinal analysis might help 
A

ROC Curve for Model Area Under the Curve $=0.6835$

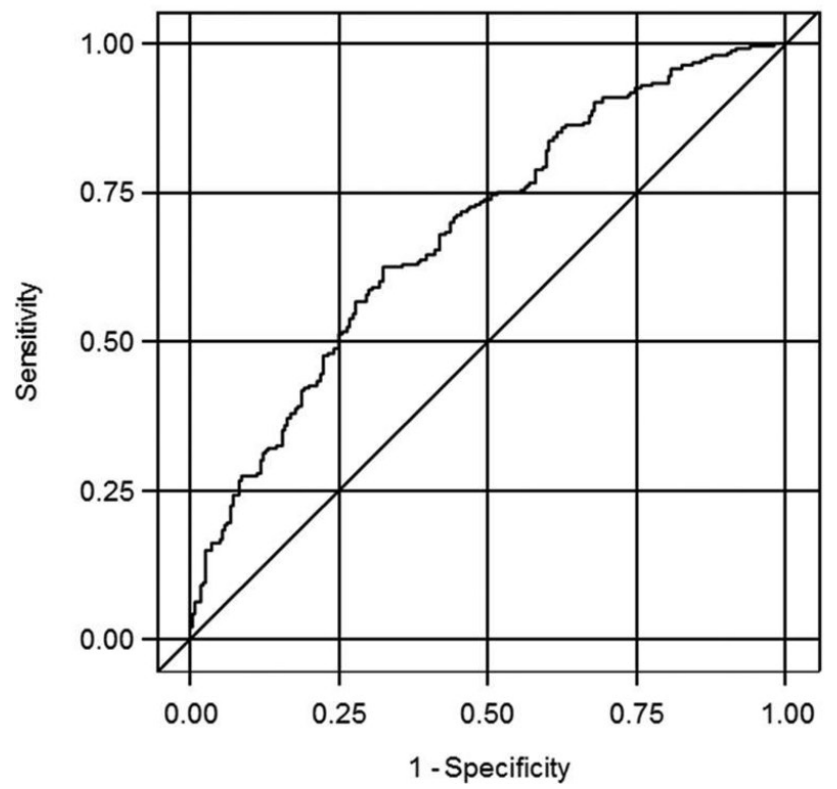

B

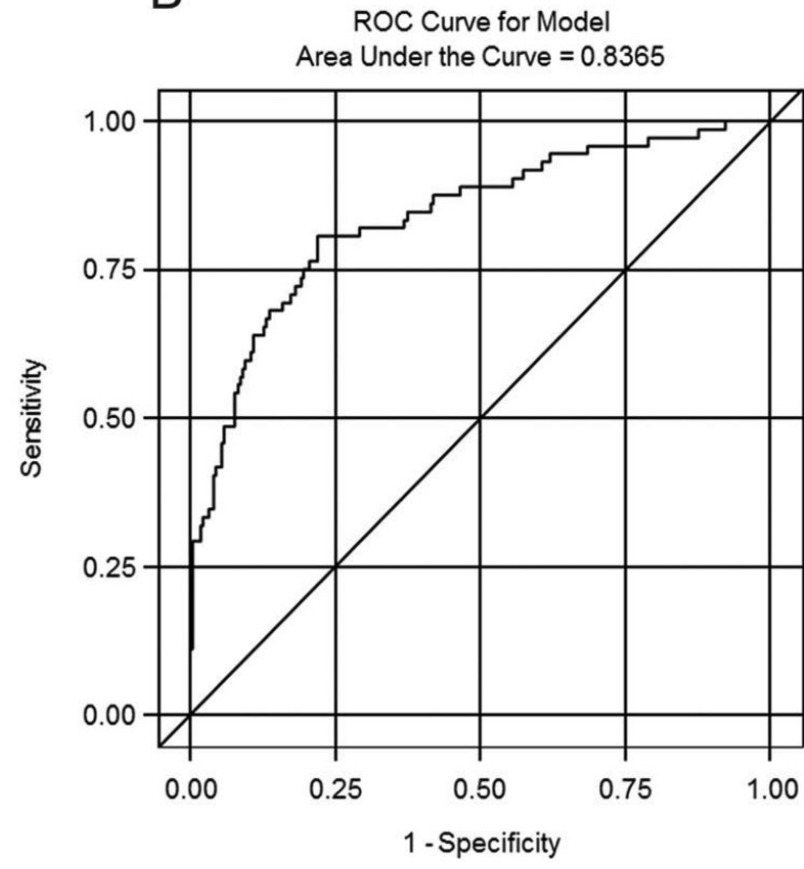

Figure 2 Receiver operating characteristic (ROC) curves from covariate-adjusted binary logistic regression with diagnosis as dependent variable and phenotype as independent variable. (A) PD-MCI versus PD-N: area under the curve (AUC) $=0.6835, \mathrm{p}<0.0001$. (B) PD-D versus PD-N: AUC $=0.8365$, $\mathrm{p}<0.0001$. $P$ values were calculated by the $\mathrm{X}^{2}$ test. PD-D, Parkinson's disease with dementia; PD-MCl, Parkinson's disease with mild cognitive impairment.

determine motor changes, as there is a great need to understand the subtypes and individual progression.

This study has also some limitations: First, the small and unequal group sizes of the different phenotypes that might have confounded the statistical analyses, however depicting real-life distribution. Second, the PD-MCI diagnosis was not based on the Movement Disorder Society (MDS) criteria, ${ }^{40}$ as these were not yet available at study initiation. Third, this study is of cross-sectional nature, thus predictions about distinct progressions of motor phenotypes need to be addressed in analyses of the longitudinal visits of the LANDSCAPE cohort with the aim to identify markers to slow down disease progression. Finally, we do not report any imaging data to investigate potential neural correlates as there are only subsamples and we do not consider the role of PD depression in this analysis. Besides that, the greatest strength is the large sample size.

In summary, changes in executive function, as well as attention/speed of processing and verbal fluency seem to be prevalent in PD and differ in respect to the different motor phenotypes, contributing to a first study of the ideal subtyping of cognitive degeneration in the PD research field. The present results show that akinetic-rigid patients undergo greater cognitive decline in several cognitive domains compared with TR-D patients, calling for early diagnosis in particular in the higher risk group and individualised therapy interventions at the earliest point.

\section{Author affiliations}

'Department of Neurology, RWTH Aachen University, Pauwelsstraße 30, Aachen, Germany

${ }^{2}$ JARA-Brain Institute Molecular Neuroscience and Neuroimaging,

Forschungszentrum Jülich GmbH and RWTH Aachen University, Aachen, Germany ${ }^{3}$ Coordinating Center for Clinical Trials, Philipps-University of Marburg, KKS Marburg, Marburg, Germany

${ }^{4}$ Medical Psychology | Neuropsychology and Gender Studies \& Center for Neuropsychological Diagnostics and Intervention (CeNDI), University Hospital Cologne, Cologne, Germany
${ }^{5}$ Division of Neurodegenerative Diseases, Department of Neurology, Technische Universität Dresden, Dresden, Germany

${ }^{6}$ Department of Neurology, University of Rostock, Rostock, Germany

${ }^{7}$ German Center of Neurodegenerative Diseases and Hertie Institute for Clinical Brain Research (DZNE), Tübingen, Germany

${ }^{8}$ Department of Neurology, Christian Albrecht University, Kiel, Germany

${ }^{9}$ Department of Neurology, Philipps University Marburg, Marburg, Germany

${ }^{10}$ Department of Geriatric Medicine, University Hospital Essen, Essen, Germany

${ }^{11}$ Department of Neurology, J.W. Goethe University, Frankfurt/Main, Germany

${ }^{12}$ Research Center Neurosensory Science, Department of Neurology, School of

Medicine and Health Sciences - European Medical School,University Oldenburg, Carl von Ossietzky University Oldenburg, Oldenburg, Germany

${ }^{13}$ Paracelsus-Elena Clinic, Centre of Parkinsonism and Movement Disorders, Department of Neurology (BM) and Department of Neurosurgery (CT), Paracelsus-

Elena Clinic, University Medical Center Goettingen, Kassel, Germany

${ }^{14}$ Department of Neurology, University Hospital Bonn, and German Center for Neurodegenerative Diseases (DZNE), Bonn, Germany

${ }^{15}$ Institute of Clinical Psychology and Psychotherapy, Technische Universität Dresden, Dresden, Germany

${ }^{16}$ Department of Psychiatry and Psychotherapy, Ludwig-Maximilians-Universität, München, München, Germany

${ }^{17}$ Department of Clinical Epidemiology, Leibniz Institute for Prevention Research and Epidemiology, Bremen, Germany

Acknowledgements The authors thank all the patients participating in the study and all the staff members in the participating centres who contributed to the study.

Contributors Manuscript: Writing of the first draft: JW; Review and critique: all other authors. Research project: Conception, organisation and execution: all authors except JW, PN, JD. Statistical analysis: Design: JW, IAH, PN, KR, JD; Execution: JW, $I A H, P N, K R, J D$.

Funding The DEMPARK study was funded by an unrestricted grant from Novartis and a grant from the International ParkinsonFonds (Deutschland) GmbH (IPD). The continuation of the study (LANDSCAPE) is funded by the German Ministry for Education and Research (BMBF 01GI1008C). KR was funded by the German Federal Ministry of Education and Research (BMBF 01GQ1402).

Competing interests GD has received lecture fees from Boston Scientific and has been serving as a consultant for Boston Scientific. He received royalties from Thieme Publishers. He is a government employee and receives through his institution funding for his research from the German Research Council, the German Ministry of Education and Research, and Medtronic. KW received reimbursement of congress 
fees from BIAL and Desitin; and grants from the Federal Ministry of Education and Research and the German Research Foundation.

Patient consent Not required.

Ethics approval The study was conducted in compliance with the Helsinki Declaration (1997; https://www.wma.net). The study protocol was approved by the Ethics Committee of the Philipps University Marburg (approval number 178/07) in March 2009 and subsequently by the local ethics committees of the participating hospitals.

Provenance and peer review Not commissioned; externally peer reviewed.

\section{REFERENCES}

1 Lees AJ, Smith E. Cognitive deficits in the early stages of Parkinson's disease. Brain 1983;106(Pt 2):257-70.

2 Lang AE, Obeso JA. Challenges in Parkinson's disease: restoration of the nigrostriata dopamine system is not enough. Lancet Neurol 2004;3:309-16.

3 Aarsland D, Andersen K, Larsen JP, et al. Prevalence and characteristics of dementia in parkinson disease: an 8-year prospective study. Arch Neurol 2003;60:387-92.

4 Rottschy C, Kleiman A, Dogan I, et al. Diminished activation of motor workingmemory networks in Parkinson's disease. PLoS One 2013;8:e61786.

5 Kudlicka A, Clare L, Hindle JV. Executive functions in Parkinson's disease: systematic review and meta-analysis. Mov Disord 2011:26:2305-15.

6 Johnson AR, Bucks RS, Kane RT, et al. motor subtype as a predictor of future working memory performance in idiopathic Parkinson's Disease. PLoS One 2016;11:e0152534-15.

7 Benninger DH, Thees S, Kollias SS, et al. Morphological differences in parkinson's disease with and without rest tremor. J Neurol 2009;256:256-63.

8 Burn DJ, Rowan EN, Allan LM, et al. Motor subtype and cognitive decline in Parkinson's disease, Parkinson's disease with dementia, and dementia with Lewy bodies. J Neurol Neurosurg Psychiatry 2006;77:585-9.

9 Poletti M, Frosini D, Pagni C, et al. Mild cognitive impairment and cognitive-motor relationships in newly diagnosed drug-naive patients with Parkinson's disease. J Neurol Neurosurg Psychiatry 2012;83:601-6.

10 Amboni M, Barone P, Hausdorff JM. Cognitive contributions to gait and falls: evidence and implications. Mov Disord 2013;28:1520-33.

11 Costa A, Peppe A, Dell'Agnello G, et al. Dopamine and cognitive functioning in de novo subjects with Parkinson's disease: effects of pramipexole and pergolide on working memory. Neuropsychologia 2009;47:1374-81.

12 Nutt JG. Motor subtype in Parkinson's disease: Different disorders or different stages of disease? Mov Disord 2016:31:957-61.

13 Alves $\mathrm{G}$, Larsen JP, Emre M, et al. Changes in motor subtype and risk for incident dementia in Parkinson's disease. Mov Disord 2006:21:1123-30.

14 Balzer-Geldsetzer M, Costa AS, Kronenbürger M, et al. Parkinson's disease and dementia: a longitudinal study (DEMPARK). Neuroepidemiology 2011:37:168-76.

15 Hughes AJ, Daniel SE, Kilford L, et al. Accuracy of clinical diagnosis of idiopathic Parkinson's disease: a clinico-pathological study of 100 cases. J Neurol Neurosurg Psychiatry 1992;55:181-4.

16 McKeith I. Dementia with Lewy bodies. In: Handbook of clinical Neurology. . Elsevier, 2007: 84. 531-48.

17 Petersen RC. Mild cognitive impairment as a diagnostic entity. J Intern Med 2004:256:183-94.

18 Emre $M$, Aarsland D, Brown R, et al. Clinical diagnostic criteria for dementia associated with Parkinson's disease. Movement Disorders 2007:22:1689-707.
19 Dubois B, Burn D, Goetz C, et al. Diagnostic procedures for Parkinson's disease dementia: recommendations from the movement disorder society task force. Mov Disord 2007;22:2314-24

20 Cummings JL, Mega M, Gray K, et al. The Neuropsychiatric Inventory: comprehensive assessment of psychopathology in dementia. Neurology 1994;44:2308-14.

21 Sheikh II, Yesavage JA. Geriatric Depression Scale (GDS): recent evidenceand development of a shorter version. Clinical gerontology: a guide to Assessment andIntervention. NY: The Haworth Press, 1986:165-73.

22 Peto V, Jenkinson C, Fitzpatrick R, et al. The development and validation of a short measure of functioning and well being for individuals with Parkinson's disease. Qual Life Res 1995:4:241-8.

23 Kalbe E, Calabrese P, Kohn N, et al. Screening for cognitive deficits in parkinson's disease with the parkinson neuropsychometric dementia assessment (PANDA) instrument. Parkinsonism Relat Disord 2008;14:93-101.

24 Bäumler G. Farb-Wort-Interferenztest (FWIT) after J. R. Stroop. Hogrefe: Göttingen 1985.

25 Tombaugh TN. Trail Making Test A and B: normative data stratified by age and education. Arch Clin Neuropsychol 2004;19:203-14.

26 Schretlen D. Brief Test of Attention (BTA). Florida: psychological assessment resources, 1997.

27 Aebi C. Validierung der neuropsychologischen Testbatterie CERAD-NP: eine MultiCenter Studie.PhD thesis: University of Basel, 2002.

28 Lineweaver TT, Bond MW, Thomas RG, et al. A normative study of Nelson's (1976) modified version of the Wisconsin Card Sorting Test in healthy older adults. Clin Neuropsychol 1999;13:328-47.

29 Sturm W, Willmes K, Horn W. Leistungsprüfsystem für 50-90jährige (LPS 50+). Hogrefe: Göttingen, 1993.

30 Stebbins GT, Goetz CG, Burn DJ, et al. How to identify tremor dominant and postural instability/gait difficulty groups with the movement disorder society unified Parkinson's disease rating scale: comparison with the unified Parkinson's disease rating scale. Mov Disord 2013;28:668-70.

31 Mukaka MM. Statistics corner: A guide to appropriate use of correlation coefficient in medical research. Malawi Med J 2012:24:69-71.

32 Arie L, Herman T, Shema-Shiratzky S, et al. Is cognitive decline similar among parkinson's disease motor sub-types? A prospective study. Innov Aging 2017;1(suppl_1):1252.

33 Fengler S, Liepelt-Scarfone I, Brockmann K, et al. Cognitive changes in prodromal Parkinson's disease: a review. Mov Disord 2017;32:1655-66.

34 Aarsland D, Bronnick K, Williams-Gray C, et al. Mild cognitive impairment in Parkinson disease: a multicenter pooled analysis. Neurology 2010;75:1062-9.

35 Kehagia AA, Barker RA, Robbins TW. Cognitive impairment in Parkinson's disease: the dual syndrome hypothesis. Neurodegener Dis 2013;11:79-92.

36 Williams-Gray CH, Evans JR, Goris A, et al. The distinct cognitive syndromes of Parkinson's disease: 5 year follow-up of the CamPalGN cohort. Brain 2009;132):2958-69.

37 Butters N, Granholm E, Salmon DP, et al. Episodic and semantic memory: a comparison of amnesic and demented patients. J Clin Exp Neuropsychol 1987:9:479-97.

38 Nieuwboer A, De Weerdt W, Dom R, et al. A frequency and correlation analysis of motor deficits in Parkinson patients. Disabil Rehabil 1998;20:142-50.

39 Fiorenzato E, Weis L, Falup-Pecurariu C, et al. MoCA vs. MMSE sensitivity as screening instruments of cognitive impairment in PD, MSA and PSP patients. Parkinsonism Relat Disord 2016:22:e59-e60.

40 Postuma RB, Berg D, Stern M, et al. MDS clinical diagnostic criteria for Parkinson's disease. Mov Disord 2015:30:1591-601. 